\title{
Electrolysis of selectively patterned Vorticella with pneumatic microchambers and electrodes
}

\author{
Moeto NAGAI*, Ryoga KOHARA*, Tuhin Subhra SANTRA** and Takayuki SHIBATA* \\ *Department of Mechanical Engineering, Toyohashi University of Technology \\ 1-1 Hibarigaoka, Tenpaku-cho, Toyohashi, Aichi 444-8580, Japan \\ E-mail: nagai@me.tut.ac.jp \\ ${ }^{* *}$ Department of Engineering Design, Indian Institute of Technology Madras, \\ Chennai, Tamil Nadu 600036, India
}

Received: 25 May 2020; Revised: 25 September 2020; Accepted: 11 November 2020

\begin{abstract}
Living machines are expected to expand the limits of silicon microtechnology. Vorticella convallaria is a ciliated protozoa and a promising linear actuator. The use of Vorticella as a $\mathrm{Ca}^{2+}$-responsive actuator, in a small system, requires a reduction in the size of the membrane treatment of selectively patterned Vorticella. However, selective cell patterning and the electrolysis of cell membranes have not yet been combined; therefore, the development of a combined method is essential. The objective of this study is to develop a method for combining selective cell patterning and the electrolysis of cell membranes. A two-layer pneumatic device was fabricated in polydimethylsiloxane (PDMS), and the device and electrodes were clamped with acrylic plates. The deformation of the PDMS membrane was controlled by compressed air. The electrochemical reactions were visualized with fluorescein, and the temporal $\mathrm{pH}$ change was evaluated. Vorticella cells were introduced into a microfluidic device and were captured in chambers at a pressure of $21 \mathrm{kPa}$. They were immobilized in the chambers after incubation for $6 \mathrm{~h}$. Further, $\mathrm{OH}^{-}$was generated from the cathode by the electrolysis of water, and the bell-shaped bodies of Vorticella became spherical. This permeabilization treatment stopped both spontaneous contraction and cilia movement. The application of DC $3 \mathrm{~V}$ was sufficient for the electrolysis of Vorticella. Selective patterning and electrochemical membrane treatments are beneficial for facilitating the use of Vorticella as a Ca ${ }^{2+}$ responsive actuator in a small system.
\end{abstract}

Keywords: Selective patterning, Electrochemical treatment, Bioactuator, Pneumatic channel, Vorticella, Permeabilization

\section{Introduction}

In the future, applications such as drug delivery and autonomous parallel computing are predicted to provide improved medical treatments and to solve mathematical problems. Living machines have been used as autonomous microrobots (Carlsen and Sitti, 2014) and are expected to expand the limits of silicon (Si) microtechnology. Biomolecular motors have been applied for parallel computation (Nicolau, et al., 2016), and bacteria have been used for drug delivery (Din, et al., 2016). The cilia motion of Vorticella (Nagai, et al., 2013a) has mixed laminar flow, and Vorticella has rotated a microstructure and functioned as a linear actuator (Nagai, et al., 2019a). Volvox has been observed to stop a flow and function as a spherical microvalve (Nagai, et al., 2015), while a sperm flagella-driven micro-biorobot has also been developed (Magdanz, et al., 2013).

Among living machines, Vorticella convallaria is a ciliated protozoa and a promising linear actuator. It is composed of a bell-shaped body (zooid) with a diameter of about 30-40 $\mu \mathrm{m}$. Its stalk is $100 \mu \mathrm{m}$ in length and $2-3 \mu \mathrm{m}$ in diameter, and it anchors itself to a surface (Ryu, et al., 2017; Upadhyaya, et al., 2008). A permeabilized stalk was contracted with $\mathrm{Ca}^{2+}$ and extended with a chelating agent of glycol ether diamine tetra acetic acid (EGTA) (Katoh and Naitoh, 1994; Ochiai, et al., 1979). The contraction/extension cycle of the Vorticella stalk was repeated over 100 times (Asai, et al., 1978), and the actuation cycle was demonstrated in a microfluidic channel (Nagai, et al., 2010; Nagai, et al., 2013b). 
The use of Vorticella as a $\mathrm{Ca}^{2+}$-responsive actuator in a small system requires a reduction in the size of the membrane treatment of selectively patterned Vorticella. To control the actuation of a Vorticella stalk via the surrounding concentration of $\mathrm{Ca}^{2+}$, the cell membrane of Vorticella is partially removed using the surfactant, saponin. Chemical permeabilization is simple (Jamur and Oliver, 2010; Shehadul Islam, et al., 2017); however, one disadvantage is that a liquid tank, valve, and pump are required for liquid exchange (Nagai, et al., 2010; Nagai, et al., 2013b), which is problematic when reducing the system's size. In previous studies, hydroxyl ions and detergent were used to permeabilize the cell membrane; hydroxide ions were generated by a low DC voltage, and the cell membrane was lysed in a microfluidic chip (Di Carlo, et al., 2005; Lee, et al., 2010; Nevill, et al., 2007). A low DC voltage has the potential to reduce the size of a system for cell lysis. Single cells were patterned at specific locations with pneumatic microchambers (Hümmer, et al., 2016), and a pneumatic valve (Nagai, et al., 2013a) and magnetic force (Nagai, et al., 2019b) were utilized to position Vorticella in a microfluidic channel. To date, cell patterning and the electrical lysis of cell membranes have only been studied separately; therefore, it is necessary to develop a combined method.

The current research developed a technique for combining selective cell patterning and the electrolysis of cell membranes. For selective cell patterning, pneumatic chambers were selected because they are available for a wide variety of cells. Vorticella was selectively patterned in pneumatic chambers, and its cell membranes were lysed by the electrolysis of water. A device for the patterning and electrochemical permeabilization of Vorticella was fabricated. The authors designed a fluidic channel and an electrode for immobilizing Vorticella to target specific areas. A DC voltage was applied to a pair of electrodes, and the $\mathrm{pH}$ change was observed using fluorescent dyes. Multiple cells of Vorticella were placed in a microfluidic channel using valves in a two-layer flow channel. Vorticella was electrochemically treated, and the changes in its shape and motion were observed.

\section{Experimental methods}

\subsection{Design of pneumatic microchambers integrated with electrodes}

This study developed a mechanism to place $V$. convallaria cells between electrodes, and then permeabilize them through electrochemical treatment (Fig. 1). The location of Vorticella was controlled to enable its use as a bioactuator. The bottom polydimethylsiloxane (PDMS) layer was used as a flow channel into which Vorticella was introduced (Fig. 1a). The top PDMS layer functioned as a pneumatic channel for applying pressure to the thin membrane. The thin PDMS membrane functioned as a valve; it was depressed by pneumatic air pumped through the pneumatic channel, and a chamber was formed Fig. 1b). Vorticella cells were maintained in the chamber, where they adhered to the surfaces and produced stalks (Fig. 1c). After the valve was opened, electrochemical treatment was conducted to permeabilize the Vorticella cell membranes (Fig. 1d).

The authors prepared a device for the electrochemical treatment of selectively patterned Vorticella (Fig. 2). Table 1 presents the major dimensions of the PDMS chambers and electrodes. To trap Vorticella cells, the height of the flow channel was set at $30 \mu \mathrm{m}$, and a total of nine chambers were arranged along a straight flow channel (Fig. 2c). A $\varphi$ 240$\mu \mathrm{m}$ chamber was designed to accomodate approximately 10 cells, while a few Vorticella cells were kept in a $\varphi 120-\mu \mathrm{m}$ chamber. Four electrodes were designed in the straight flow channel (Fig. 2a); the two left electrodes were the cathode,

(a)

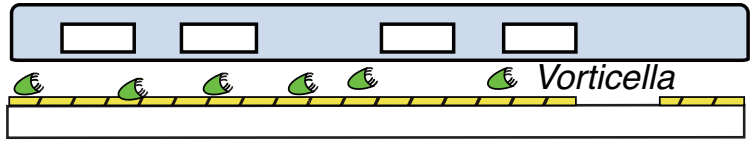

(c)

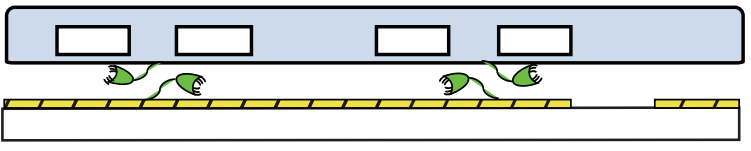

(b)

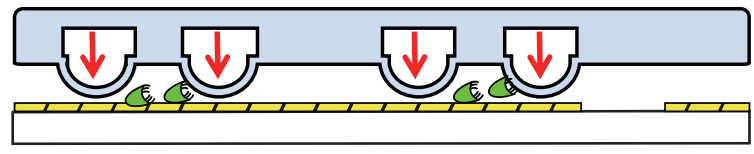

(d)

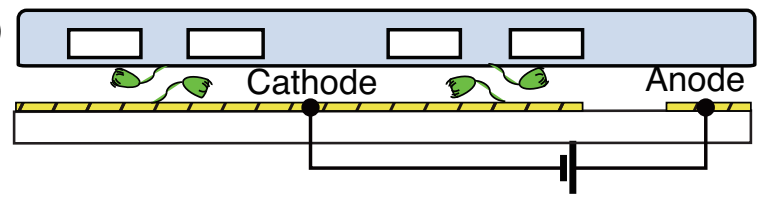

Fig. 1 Schematic of a device for the electrochemical treatment of selectively placed Vorticella cells. (a) Introduction of Vorticella to a channel. (b) Chamber formation by the deformation of the pneumatic valve. (c) Spontaneous adhesion of Vorticella to the channel's surface. (d) Electrochemical treatment of the Vorticella cells. 


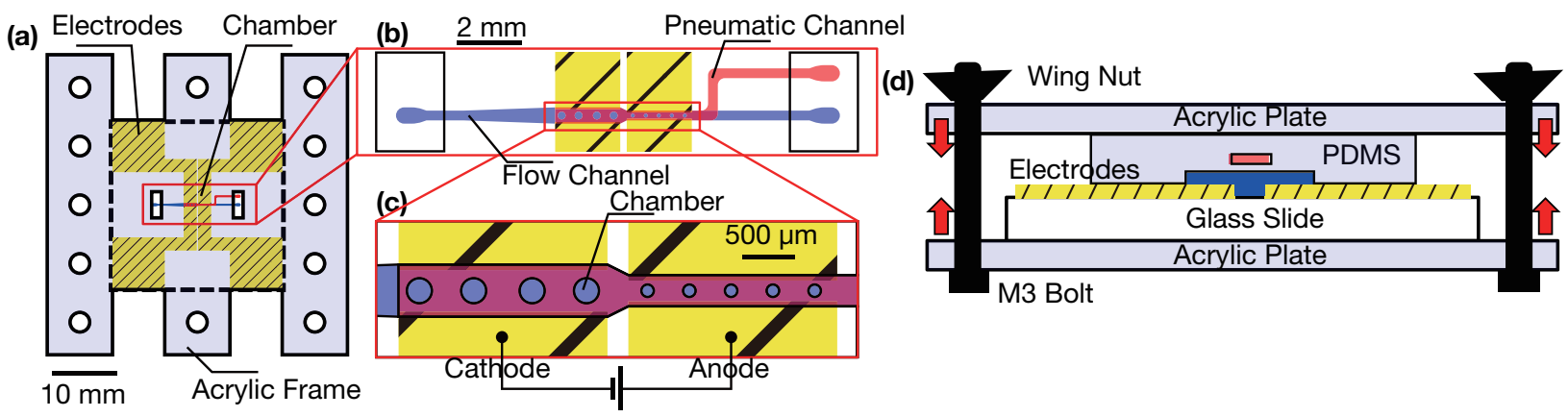

Fig. 2 Schematic of a PDMS chip and electrode substrate clamped with a pair of acrylic plates. (a) Top view of the entire chip. (b) Entire view of flow channels and a partial view of four electrodes. (c) Enlarged view of four electrodes and nine chambers along a channel. (d) Cross-sectional view of a clamp, PDMS chip, and glass slide.

Table 1 Dimensions of PDMS chambers and electrodes.

\begin{tabular}{l|l|l}
\hline \hline Channel width & Electrode width & $\begin{array}{l}\text { Chamber } \\
\text { diameter }\end{array}$ \\
\hline $500 \mu \mathrm{m}$ & $400 \mu \mathrm{m}$ & $240 \mu \mathrm{m}$ \\
\hline $300 \mu \mathrm{m}$ & $200 \mu \mathrm{m}$ & $120 \mu \mathrm{m}$ \\
\hline
\end{tabular}

and the two right electrodes were the anode (Fig. 2c). For the ease of connection of wires, square electrode pads of size $8 \times 8$-mm were used. The gaps between the up and down electrodes were $200 \mu \mathrm{m}$ for the $300-\mu \mathrm{m}$ wide channel and 500 $\mu \mathrm{m}$ for the $400-\mu \mathrm{m}$ wide channel.

\subsection{Fabrication of pneumatic microchambers integrated with electrodes}

\subsubsection{Preparation of SU-8 mold}

The bottom and top microchannels were molded from SU-8 molds, which were prepared in SU-8 3050 photoresist (Nippon Kayaku Co., Ltd.) by using photolithography (Fig. 3a, c). First, a 3-inch Si wafer was cleaned with acetone and isopropyl alcohol (IPA). The Si substrate was spin coated with SU-8 at 3,000 rpm for $60 \mathrm{~s}$ using a spin coater (1H-DX2, Mikasa Corp.). For the introduction of Vorticella, the target thickness was set to $30 \mu \mathrm{m}$. The wafer was pre-baked at $65^{\circ} \mathrm{C}$ for $2.5 \mathrm{~min}$, at $95^{\circ} \mathrm{C}$ for $20 \mathrm{~min}$, and at $65^{\circ} \mathrm{C}$ for $2.5 \mathrm{~min}$. A two-stage heating and cooling was employed to reduce the difference in thermal expansion between the SU-8 and the wafer. The SU-8 film was exposed in contact mode for $15 \mathrm{~s}$ via a film mask with a double-sided mask aligner (PEM-800, Union Optical Co., Ltd.). The amount of integrated light was $200 \mathrm{~mJ} / \mathrm{cm}^{2}$. The SU-8 was post-baked at $65^{\circ} \mathrm{C}$ for $9 \mathrm{~min}$, at $95^{\circ} \mathrm{C}$ for $5 \mathrm{~min}$, and at $65^{\circ} \mathrm{C}$ for $2.5 \mathrm{~min}$. It was developed

(a)

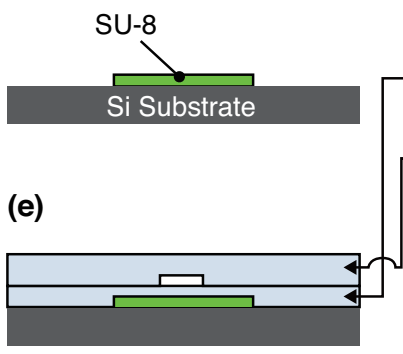

(b)

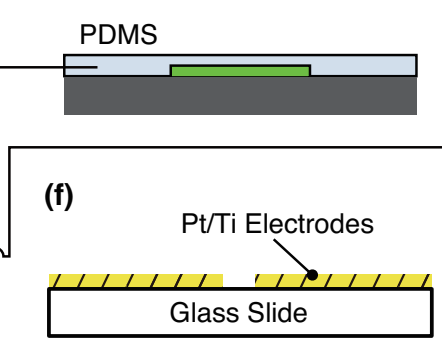

(c)

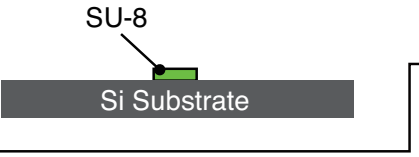

(g)

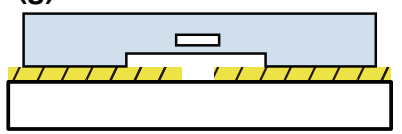

(d)

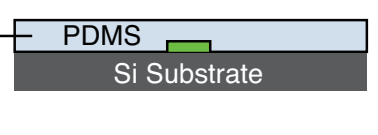

Fig. 3 Fabrication process of a two-layer microfluidic device using thin PDMS film and the integration process of the device and electrodes. (a) SU-8 mold fabrication and (b) PDMS molding for flow channel. (c) SU-8 mold fabrication and

(d) PDMS molding for pneumatic channel. (e) Bonding of the bottom and top layers. (f) Patterning of platinum (Pt)/ titanium (Ti) electrodes. (g) Integration of the PDMS on the electrodes. 
in 2-methoxy-1-methylethyl acetate (Wako Pure Chemical Industries, Ltd.) and rinsed with acetone for $10 \mathrm{~min}$. The surface of the flow channel mold was treated in a vapor of trichloro $(1 \mathrm{H}, 1 \mathrm{H}, 2 \mathrm{H}, 2 \mathrm{H}$-perfluorooctyl) silane (Sigma Aldrich), which was vaporized at $80^{\circ} \mathrm{C}$ for 2 min to enhance the releasability of PDMS from the SU-8 mold.

\subsubsection{Fabrication of top and bottom polydimethylsiloxane layers}

The top and bottom layers were replicated in PDMS (Sylgard 184, Dow Corning) from the SU-8 molds by soft lithography (Fig. 3b, d). The main PDMS agent and a curing agent were mixed at a ratio of 10:1, and air bubbles in the PDMS were removed with an aspirator (Dry Aspirator DAS-01, As One Corporation). Degassed PDMS was poured over the flow channel mold with a height of $30 \mu \mathrm{m}$ and was spin coated at 1,500 rpm for $60 \mathrm{~s}$. The wafer was maintained at room temperature for $10 \mathrm{~min}$ to flatten the PDMS. It was then cured at $50^{\circ} \mathrm{C}$ for $1 \mathrm{~h}$ on a hotplate (RSH-1 DR, As One Corporation) and again at $80^{\circ} \mathrm{C}$ for $1 \mathrm{~h}$ (Fig. 3b). Degassed PDMS was also poured over the top SU-8 mold, and air bubbles in the PDMS were removed with the aspirator. The PDMS was cured at $80^{\circ} \mathrm{C}$ for 40 min on the hotplate (Fig. $3 \mathrm{~d}$ ). The cured PDMS was released using a scalpel and tweezers and was shaped with a razor. Inlets and outlets of 0.5 $\mathrm{mm}$ were punched in the PDMS chip under a stereomicroscope (SZ61, Olympus Corporation).

The PDMS chip was then ultrasonically cleaned (VS-100III, As One Corporation) at $45 \mathrm{kHz}$ in deionized (DI) water and was dehydrated on the hot plate at $80^{\circ} \mathrm{C}$. Both PDMS surfaces were treated with the corona treater (BD20AC, ElectroTechnic Products, Inc.) for $40 \mathrm{~s}$ (Haubert, et al., 2006). The top PDMS chip was then aligned with the bottom layer, and the assembled chip was subjected to light finger pressure (Fig. 3e). After baking at $80^{\circ} \mathrm{C}$ for $1 \mathrm{~h}$, the bonded PDMS chip was released from the SU-8 mold.

\subsubsection{Preparation of platinum/titanium electrodes}

A glass slide $(76 \times 26 \times 1 \mathrm{~mm}$, white, No. 1, Matsunami Glass Industry Co., Ltd.) was cut into three equal parts (each $26 \times 26 \mathrm{~mm}$ ). It was cleaned with a mixture of hydrogen peroxide and sulfuric acid at a ratio of 1:3. The glass slide was dehydrated at $140^{\circ} \mathrm{C}$ for $5 \mathrm{~min}$. Hexamethyldisilazane was spin coated on the glass surface at 3,000 rpm for $60 \mathrm{~s}$, and it was baked at $110^{\circ} \mathrm{C}$ for $90 \mathrm{~s}$. A positive resist (OFPR-8600, $52 \mathrm{cp}$ ) was spin coated onto the glass slide at 3,000 rpm for $60 \mathrm{~s}$ and was pre-baked at $110^{\circ} \mathrm{C}$ for $90 \mathrm{~s}$. The resist was exposed in contact mode for $20 \mathrm{~s}$ (integral amount of light: 250 $\mathrm{mJ} / \mathrm{cm}^{2}$ ). The photoresist was then developed in NMD-3 (Wako Pure Chemical Industries, Ltd.) for 10 min. The wafer was rinsed with DI water and was post-baked at $140^{\circ} \mathrm{C}$ for $5 \mathrm{~min}$. Thin $\mathrm{Ti}$ and $\mathrm{Pt}$ films were deposited on the patterned glass via a sputtering apparatus (L-250S-FH, Anelva Co., Ltd.). Target film thicknesses of $10 \mathrm{~nm}$ for Ti and $100 \mathrm{~nm}$ for $\mathrm{Pt}$ were applied. The Pt/Ti films were removed in acetone for $10 \mathrm{~min}$ by ultrasonic cleaning at $100 \mathrm{kHz}$ (Fig. $3 \mathrm{f}$ ). The surface was cleaned with IPA and was blown with nitrogen.

\subsubsection{Integration of the polydimethylsiloxane pneumatic chamber and electrodes with an acrylic clamping jig}

The PDMS flow channel and electrode substrate were clamped with an acrylic jig and M3 bolts (Fig. 2d and Fig. $3 \mathrm{~g})$. To enable the reuse of the electrode substrate, permanent bonding was not performed. Transparent hard-coated acrylic resin plates (Acrysunday Co., Ltd., acrylic resin cast, pencil hardness: 6H, MR2-001, thickness: $2 \pm 0.2 \mathrm{~mm}$ ) and an inverted microscope were used to observe the inside of the PDMS chip. The plates were scratch resistant and highly permeable (total light transmittance: 93\%). The plate was milled and drilled to match the size of the microfluidic device (Fig. 2a).

\subsection{Observation of fluorescence for $\mathrm{pH}$ measurement}

$\mathrm{OH}^{-}$was generated from the cathode and was measured using fluorescein (Acid yellow 73, Tokyo Chemical Industry Co., Ltd.), which is a pH-dependent fluorescent dye. Fluorescein powder was dissolved in acetone and diluted to $50 \mu \mathrm{M}$ with DI water. The state of the electrochemical reaction between the electrodes was observed using an inverted fluorescence microscope, and the intensity was measured from the image. The fluorescence intensity over the distance between the pair of electrodes was measured to evaluate the $\mathrm{pH}$ change. Based on the obtained intensity distribution, the 
electrochemical reaction between the electrodes was investigated.

For fluorescence observation, a fluorescence microscope (Ti-U, Nikon Instruments Inc.) equipped with a cooled charge-coupled device (CCD) camera (DS-Qi1Mc, Nikon Instruments Inc.) was used. Blue light was produced from a fiber light source (Intensilight C-HGFI, Nikon Instruments Inc.) via a fluorescence cube (B-2A, EX 450-490 nm, DM $505 \mathrm{~nm}$, BA $520 \mathrm{~nm}$, Nikon Instruments Inc.).

\subsection{Injection and trapping of Vorticella}

A cell suspension of $V$. convallaria was prepared according to a previously described method (Nagai, et al., 2013b; Vacchiano, et al., 1991). A culture medium was extracted from 1-g/L wheatgrass powder (Pines wheatgrass, Pines International, Inc.) by first, autoclaving at $121^{\circ} \mathrm{C}$ and second, removing the solid content by filtration. The cells were incubated in a 1 -L flask containing a medium at $20^{\circ} \mathrm{C}$ while agitating for $24 \mathrm{~h}$ to obtain floating $V$. convallaria cells.

A PDMS flow channel was filled with mineral water (Morinomizu, natural spring water, Japan Coca-Cola Co.) in a vacuum chamber. A suspension of Vorticella was introduced, and pneumatic pressure was applied from a compressor (Mini air compressor AC-500, Tow Marker Products CO., LTD.). The valve was gradually deformed to guide cells to the center of the chamber.

\subsection{Voltage power supply and observation setup for electrolysis of Vorticella}

The electrodes were connected to a DC power supply (ePS80WL, Fujitsu Access Co., LTD.), and DC voltage was applied to the pair of electrodes. The fluorescence of the dyes and the shape changes of Vorticella were observed under a microscope. Trapped Vorticella cells were electrochemically treated to permeabilize the cellular membrane with the hydroxide ion $\left(\mathrm{OH}^{-}\right)$generated from the cathode. Vorticella cells were observed with an inverted microscope (Eclipse TE2000-U, Nikon Instruments Inc.) equipped with a CCD camera (Digital Sight DS-2Mv, Nikon Instruments Inc.).

\section{Results and discussion}

\subsection{Fabrication of pneumatic microfluidic chambers}

Each PDMS layer was replicated from an SU-8 3050 mold, and the first flow layer and the second pneumatic layer were permanently bonded following plasma treatment (Fig. 4a). A PDMS membrane was formed between the first and second layer to create pneumatic valves and was used to capture and immobilize Vorticella cells in the chambers. A pneumatic channel was then properly aligned with a flow channel, and circles were placed in the center of the flow channel. Four circles $(\varphi 240 \mu \mathrm{m})$ were placed in a 500- $\mu \mathrm{m}$ wide channel and five circles $(\varphi 120 \mu \mathrm{m})$ were placed in a $300-\mu \mathrm{m}$ wide channel. A thin PDMS membrane was not formed on these circles, and the membrane enabled the formation of chambers for capturing Vorticella cells. The diameter of the $\varphi 240-\mu \mathrm{m}$ chambers for placing multiple Vorticella cells was $228.5 \pm 4.2 \mu \mathrm{m}(\mathrm{N}=4)($ Fig. $4 \mathrm{~b})$, and the diameter of the $\varphi 120-\mu \mathrm{m}$ chambers was $97.6 \pm 6.7 \mu \mathrm{m}(\mathrm{N}=5)(\mathrm{Fig}$. 4c).

A cross section of the PDMS pneumatic channel was measured (Fig. 4d, e); the height of each channel was $28.9 \mu \mathrm{m}$ $\pm 1.6 \mu \mathrm{m}(\mathrm{N}=6)$. The height of the circular parts was sufficient to bond the upper pneumatic layer to the lower PDMS flow channel layer. Using the channel height and the thickness (around $50 \mu \mathrm{m}$ ) of the PDMS that was spin coated at 1,500 rpm for $60 \mathrm{~s}$, the thickness of the PDMS membrane was estimated at $20 \mu \mathrm{m}$ (Zhang, et al., 2004). The widths of the typical PDMS membranes were approximately 80, 130, and $280 \mu \mathrm{m}$, and these widths affected the deformation of the membrane.

\section{2 $\mathrm{pH}$ measurement with fluorescein}

The fluorescence of fluorescein was measured, while $\mathrm{H}^{+}$was generated by the electrolysis of water in a flow channel (Fig. 5). A pair of electrodes with a 400- $\mu \mathrm{m}$ gap was used for simplicity. The upper electrode was the cathode, and the lower electrode was the anode. The $\mathrm{pH}$ of a solution was changed by the following electrochemical reactions.

Anode reaction:

$$
2 \mathrm{H}_{2} \mathrm{O}(\mathrm{L}) \rightarrow 4 \mathrm{H}^{+}(\mathrm{aq})+\mathrm{O}_{2}(\mathrm{~g})+4 \mathrm{e}^{-}
$$




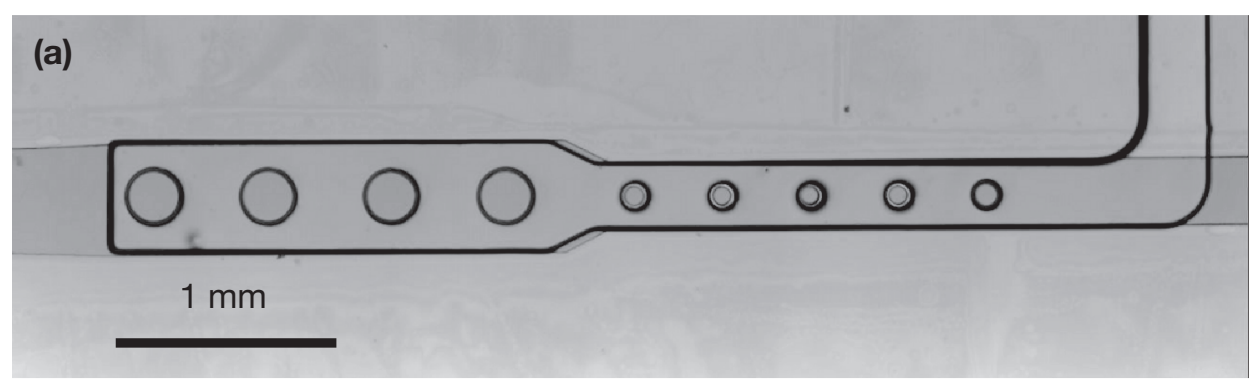

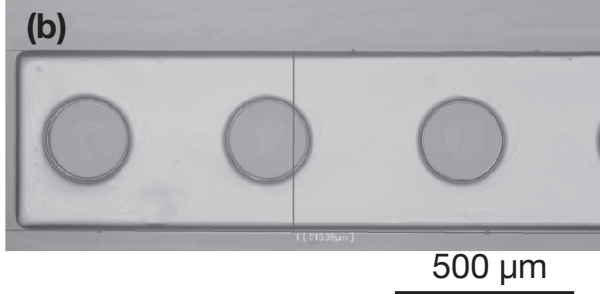

(d)

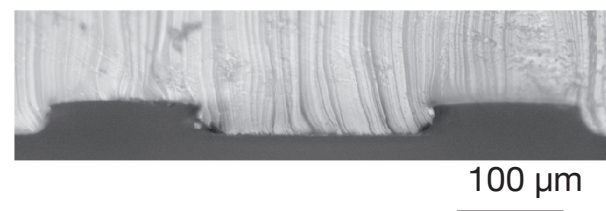

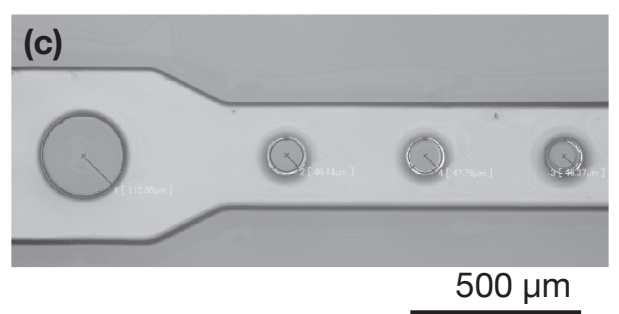

(e)

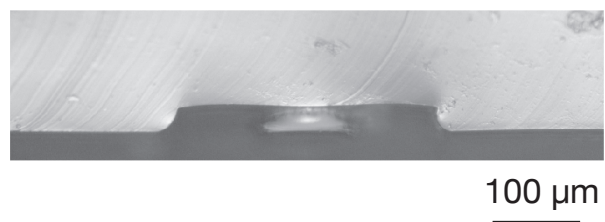

Fig. 4 Microscope images of a fabricated device. (a) A pneumatic valve device, which was composed of a two-layer flow channel. Top view of a pneumatic channel on the (b) left and (c) right sides. Cross-sectional views of the (d) 500- $\mu \mathrm{m}$ and (e) 300- $\mu \mathrm{m}$ wide PDMS pneumatic channels. $\phi 240 \mu \mathrm{m}$ and $\varphi 120 \mu \mathrm{m}$ circles were placed in the middle of $500-\mu \mathrm{m}$ and $300-\mu \mathrm{m}$ wide channels.

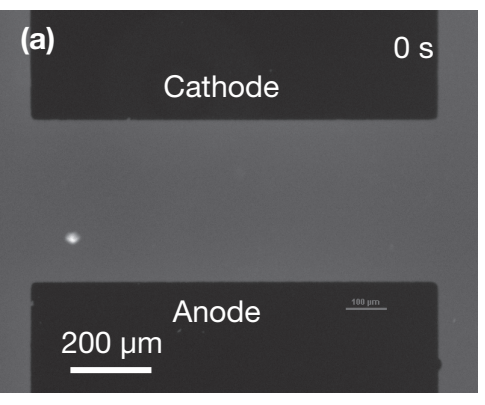

(c)

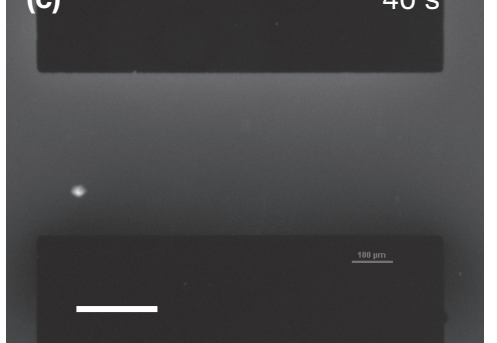

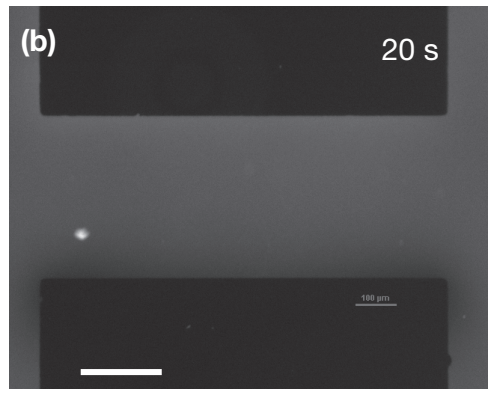
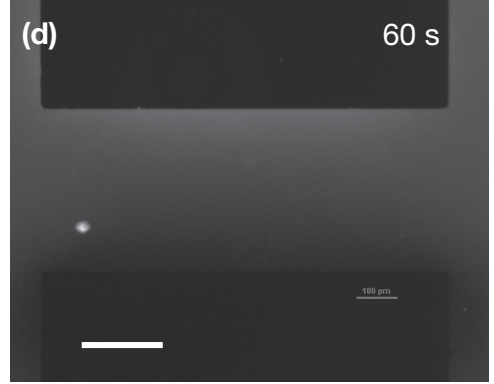

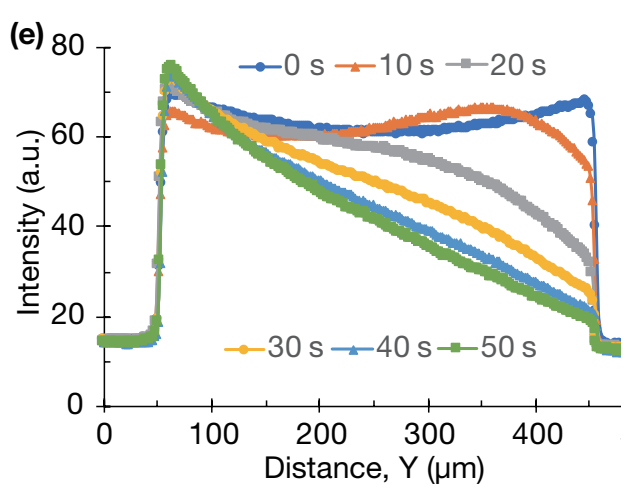

Fig. 5 Visualized hydroxide ions with fluorescein in a region between a pair of electrodes with a gap of $400 \mu \mathrm{m}$. The applied voltage was DC 2 V. (a)-(d) Time-series microscope images of fluorescein around the electrode pair. (e) Fluorescence intensity along a line connecting the electrodes.

Cathode reaction:

$$
4 \mathrm{H}_{2} \mathrm{O}(\mathrm{L})+4 \mathrm{e}^{-} \rightarrow 4 \mathrm{OH}^{-}(\mathrm{aq})+2 \mathrm{H}_{2}(\mathrm{~g})
$$

The fluorescence decreased from the end of the anode immediately after voltage application (Fig. 5a-d). The fluorescence intensity near the cathode remained or slightly increased, while the intensity near the anode remained almost 
constant (Fig. 5e). After applying voltage for $40 \mathrm{~s}$, the intensity change decelerated. The increase in the value of the applied voltage increased the amount of electrochemical reaction. A similar $\mathrm{pH}$ profile to this result was observed using pH sensitive dyes, sodium alginate, and calcium carbonate (Cheng, et al., 2011a; Cheng, et al., 2011b). This intensity change suggests that $\mathrm{H}^{+}$and $\mathrm{OH}^{-}$were generated around the anode and cathode, respectively. Hydroxide ions generated around the cathode were applied to the electrochemical treatment of Vorticella.

\subsection{Trapping Vorticella with pneumatic chambers}

A suspension of Vorticella cells $(185$ cells $/ \mu \mathrm{L})$ in a wheatgrass medium was introduced into a flow channel by hydrostatic pressure. $V$. convallaria was transported into the vicinity of a chamber by fine adjustment of the hydrostatic pressure. Pneumatic microchambers were formed by applying pneumatic pressure, which was gradually increased from $1 \mathrm{kPa}$ to prevent the cells from being caught by the PDMS membrane. Vorticella escaped through small gaps between the valves near a $240-\mu \mathrm{m}$ diameter chamber at $16 \mathrm{kPa}$, possibly due to insufficient deformation. To retain Vorticella in the chamber, the pressure was increased to $21 \mathrm{kPa}$; 9 of 10 cells were guided into a chamber with a diameter of $240 \mu \mathrm{m}$ and captured in it (Fig. 6). 1 of 10 cell was not guided into the chamber and was sandwiched between the valve membrane and the substrate. The valve functioned without being peeled off, even at a pressure of $21 \mathrm{kPa}$.

Pneumatic pressure was applied to form a $120-\mu \mathrm{m}$ chamber. Vorticella passed through the gaps between a PDMS membrane and the bottom glass substrate and cells were not captured. Since the width of the thin film around a $120-\mu \mathrm{m}$ chamber was narrower than a $240-\mu \mathrm{m}$ diameter chamber, the PDMS membrane was not sufficiently deformed at $21 \mathrm{kPa}$. A higher pressure is needed to form a $120-\mu \mathrm{m}$ chamber.

To ensure the adherence of Vorticella, pneumatic pressure was maintained at $21 \mathrm{kPa}$ for $6 \mathrm{~h}$. The valve was returned to the initial position by releasing the pneumatic pressure. Vorticella cells were selectively immobilized in the right three of the four chambers, where they grew stalks (Fig. 7). Cells were caught between the membrane around the left chamber and were pushed away from the valve (Fig. 7a). The width of the PDMS membrane around the left chamber was $80 \mu \mathrm{m}$, which was smaller than the $280 \mu \mathrm{m}$ of one of the right three chambers; hence, the valve deformation was insufficient for the selective patterning of Vorticella. Left unwanted cells shown in Fig. 7(a) did not interfere with the next experiment and they were ignored. The selective patterning method with a pneumatic valve (Hümmer, et al., 2016; Nagai, et al.,
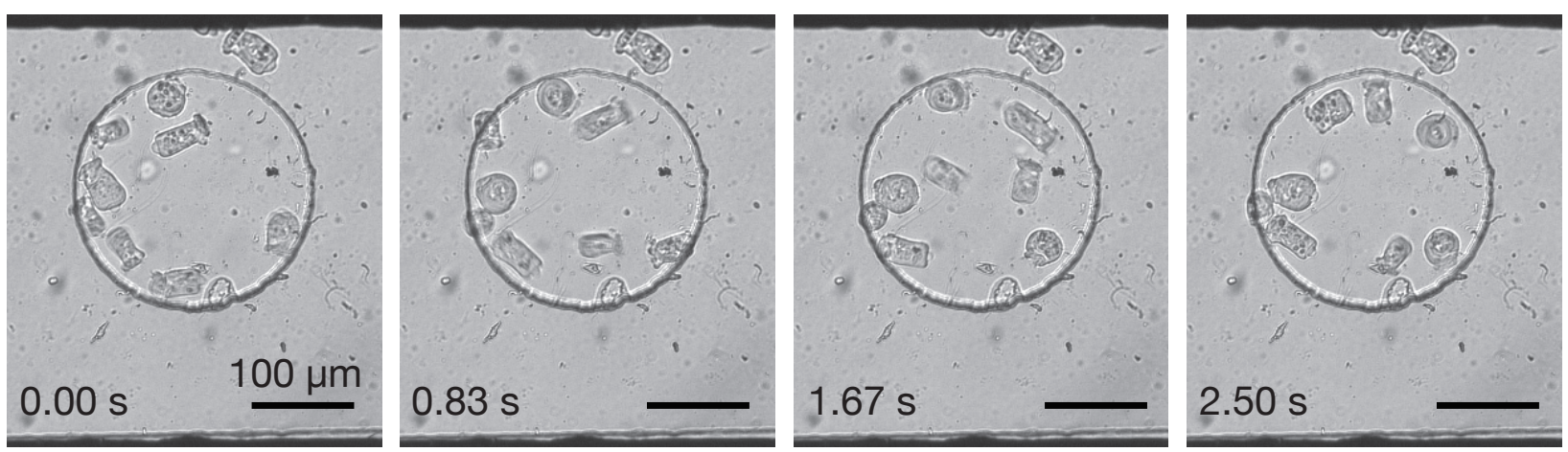

Fig. 6 Time-lapse images of nine Vorticella cells captured in a pneumatic microchamber. Some cells swam in this chamber but did not escape. One Vorticella cell was not guided to the chamber and was captured with the PDMS valve.
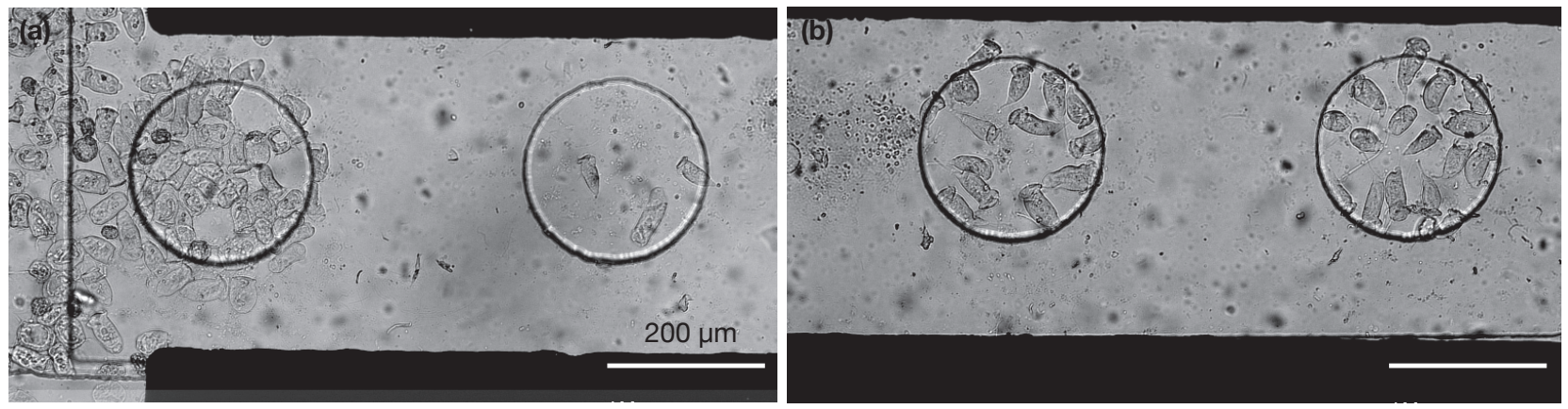

Fig. 7 Microscope images showing Vorticella cells around (a) the two left chambers and (b) the two right chambers $6 \mathrm{~h}$ after the closure of the pneumatic valves. 

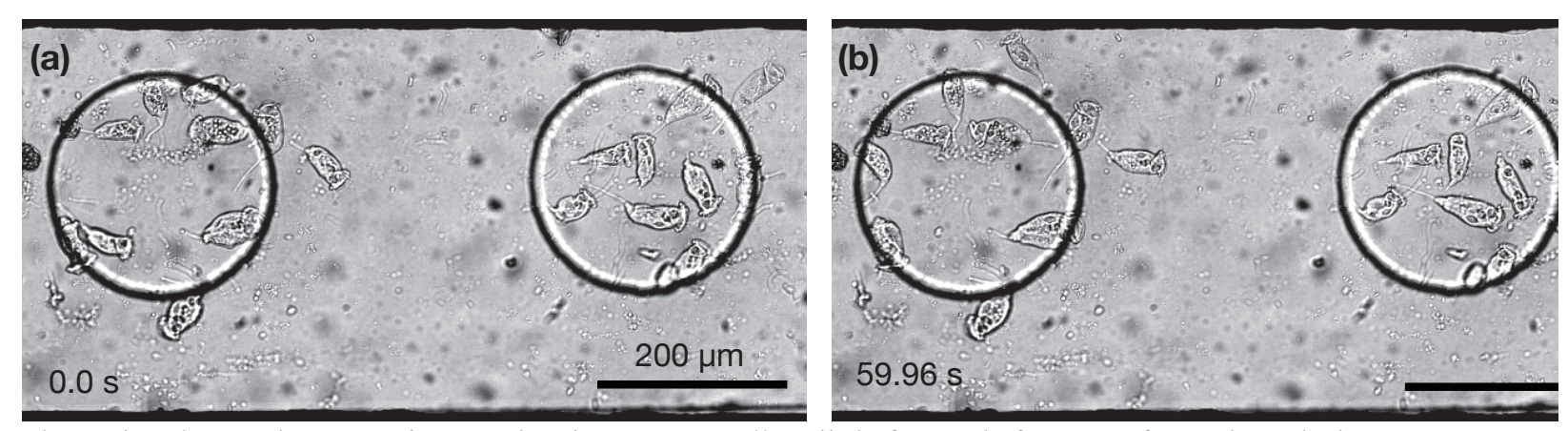

Fig. 8 Time-lapse microscopy images showing 18 Vorticella cells before and after $60 \mathrm{~s}$ of 2-V electrolysis.
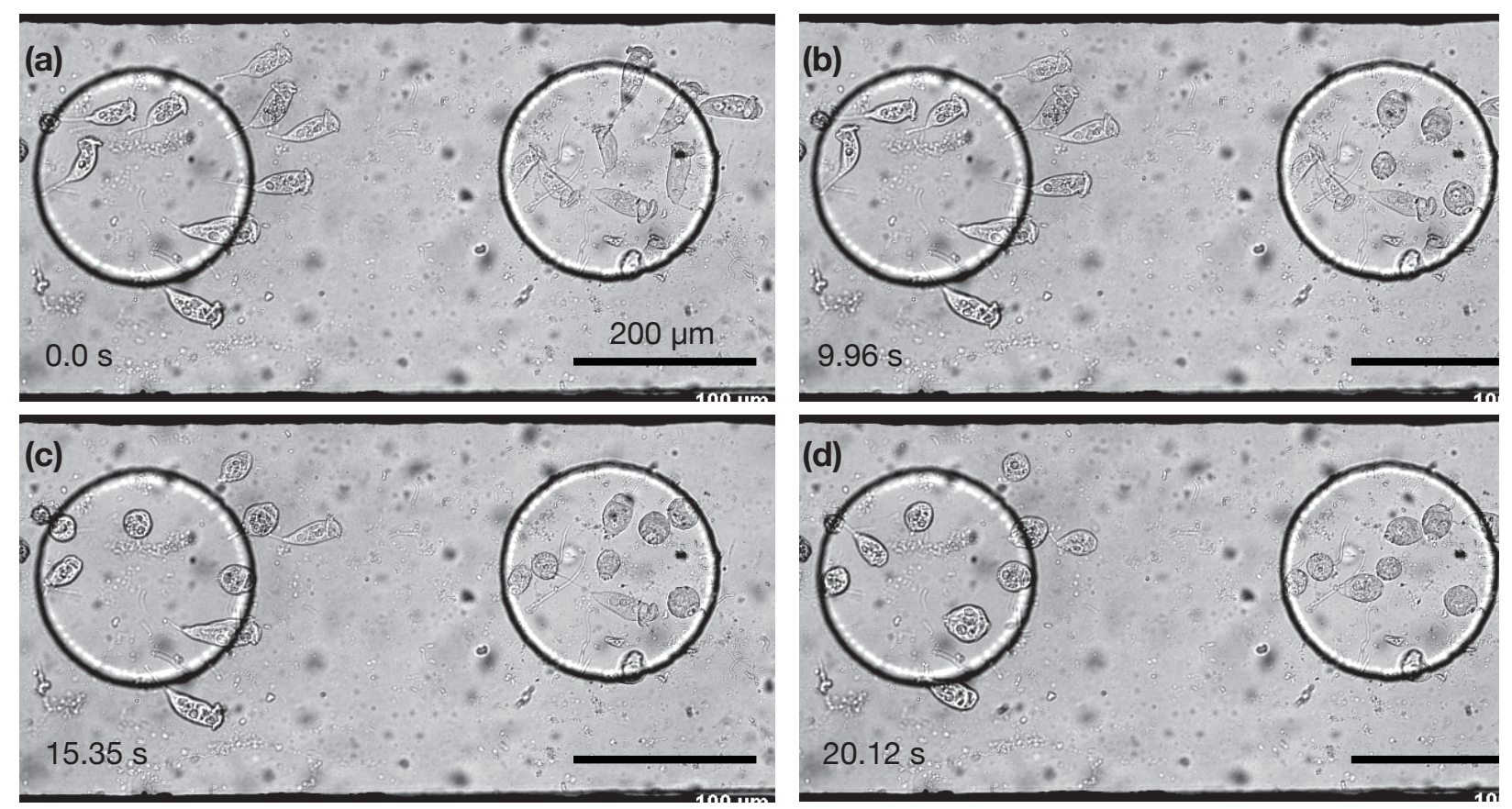

Fig. 9 Time-lapse microscopy images showing 18 Vorticella cells during $20 \mathrm{~s}$ of $3-\mathrm{V}$ electrolysis.

2013a) was expanded for the electrochemical treatment of Vorticella positioned at the central part of the channel.

\subsection{Electrochemical treatment of patterned Vorticella}

Hydroxide ions were generated by the electrolysis of water from the cathode, and the permeabilization treatment of the Vorticella cells between the electrodes was conducted. After the 6-h capture and immobilization of Vorticella, the valve was opened, and the culture solution in the channel was replaced with spring water. Vorticella cells were observed with an inverted microscope in two chambers in the flow channel; the chambers had a width of $500 \mu \mathrm{m}$ and an interelectrode distance of $400 \mu \mathrm{m}$. DC voltages of 2 and $3 \mathrm{~V}$ were applied between left and right electrodes.

After the application of DC $2 \mathrm{~V}$ to the electrodes, 18 Vorticella cells were observed. Fig. 8 contains the microscopy images of Vorticella at $0 \mathrm{~s}$ and $60 \mathrm{~s}$ after voltage application. The shape of Vorticella did not change, and the Vorticella cells were observed to spontaneously move, even after $4 \mathrm{~min}$ of voltage application. If permeabilization is successful, Vorticella stops all movement; however, there was no evidence that the cellular membranes were permeable, and it was concluded that DC $2 \mathrm{~V}$ was insufficient for the electrolysis of Vorticella.

To increase the effect of electrolysis, the voltage was increased from DC 2 to $3 \mathrm{~V}$. The 18 cells started to contract with higher frequency, and their bodies became spherical (Fig. 9). Most of the cells suddenly became spherical after spontaneous contraction. The change ratio of Vorticella bodies over time is plotted in Fig. 10(a). All Vorticella changed from a bell shape to a spherical shape at $20 \mathrm{~s}$ and did not exhibit spontaneous contraction and cilia movement. The shape changed faster on the right side of the chambers than on the left side. Fig. 10(b) shows visualized hydroxide ions in a region between left and right electrodes. The fluorescence was stronger on the side close to the anode. This fluorescence 
distribution is consistent with the faster treatment of Vorticella on the right side of the chambers. The shape change was similar to that observed with a solution of saponin, which is a detergent used for chemical permeabilization. This shape change and the cessation of spontaneous movement suggest that the cellular membrane was permeable. For further confirmation of membrane permeabilization, Vorticella was kept for $12 \mathrm{~h}$ in a channel and permeabilized at $3 \mathrm{~V}$. After the permeabilization, a stalk of Vorticella contracted in spring water containing calcium ion. Spring water was replaced with a chelating solution (EGTA $4 \mathrm{mM}, \mathrm{KCl} 100 \mathrm{mM}$, Tris maleate $50 \mathrm{mM}, \mathrm{pH} 7.0$ ) and the stalk was extended. Although electroporation potentially cause permeabilization, the electric field strength is assumed to be too low to cause electroporation. The gap between the cathode and anode was $400 \mu \mathrm{m}$, and the maximum average electric field strength was $50 \mathrm{~V} / \mathrm{cm}$ at $2 \mathrm{~V}$ and $75 \mathrm{~V} / \mathrm{cm}$ at $3 \mathrm{~V}$. These electric field strengths were relatively low to induce electroporation.

A microcomponent was rotated by the contraction of Vorticella in a microchannel (Nagai, et al., 2019b). However, a Vorticella cell contracted and elongated spontaneously and it was not responsive to $\mathrm{Ca}^{2+}$. This cell permeabilization technology can provide $\mathrm{Ca}^{2+}$-responsiveness to this actuation system. The permeabilization of Vorticella has been chemically performed in previous studies by exchanging solutions for which a solution-exchange mechanism was required (Nagai, et al., 2010; Nagai, et al., 2013b). In the current study, a DC power supply was applied for permeabilization, and a solution-exchange mechanism was omitted. This evaluation is useful for reducing the size of the permeabilization system for Vorticella.
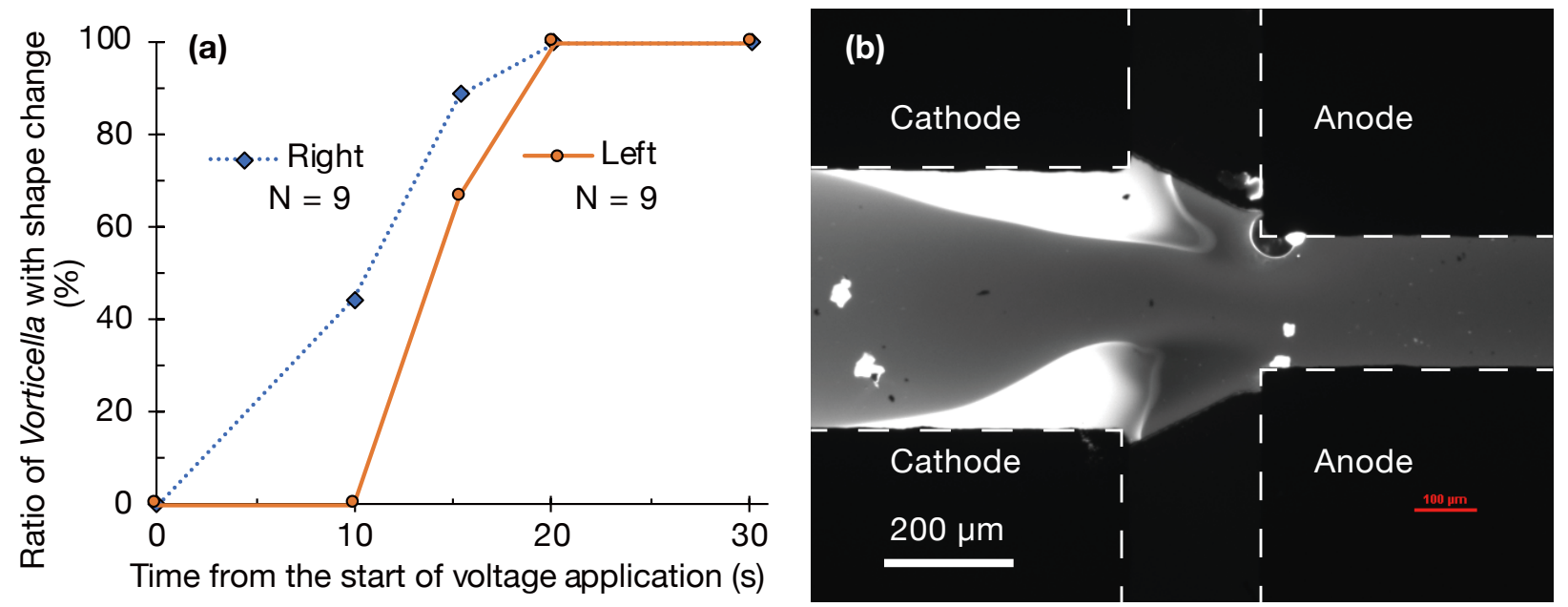

Fig. 10 (a) Ratio of $V$. convallaria with shape change over time at DC 3 V. The shapes did not change at DC 2V. (b) Visualized hydroxide ions with fluorescein in a region between left and right electrodes in flow. The left two electrodes were cathode and the applied voltage was DC $6 \mathrm{~V}$.

\section{Conclusions}

This study successfully achieved the combined selective patterning of Vorticella and its electrochemical permeabilization. A pneumatic valve with electrodes was designed and developed. A two-layer pneumatic device was fabricated, and the deformation of a PDMS membrane was controlled to capture Vorticella in chambers. Vorticella was selectively patterned with pneumatic valves, and water electrolysis was used to permeabilize the Vorticella membranes. Vorticella was captured in a chamber at a pressure of $21 \mathrm{kPa}$ and immobilized in a culture medium after $6 \mathrm{~h}$ of incubation. Further, $\mathrm{OH}^{-}$was generated from the cathode by DC 3-V electrochemical reactions, and the bodies of Vorticella changed from a bell shape to a spherical shape within $20 \mathrm{~s}$. The electrochemical reaction was also visualized with fluorescein, and the temporal change of the $\mathrm{pH}$ was evaluated to confirm the generation of hydroxyl ions. The permeability treatment stopped all spontaneous contraction and cilia movement. It was demonstrated that the electrical approach was able to remove the mechanical parts required for solution exchange.

The authors' future work will involve reducing the size of the entire system and developing microdevices incorporating microorganisms while removing the fluidic exchange system. A 9-V battery has previously been used to reduce the size of a microfluidic system (Addae-Mensah, et al., 2010). This concept can be applied to the objective of downsizing an entire system and could lead to the practical application of Vorticella-derived $\mathrm{Ca}^{2+}$-responsive linear 
actuators.

\section{References}

Addae-Mensah, K. A., Cheung, Y. K., Fekete, V., Rendely, M. S. and Sia, S. K., Actuation of elastomeric microvalves in pointof-care settings using handheld, battery-powered instrumentation, Lab on a Chip, Vol.10, No.12 (2010), pp.1618-1622.

Asai, H., Ochiai, T., Fukui, K., Watanabe, M. and Kano, F., Improved Preparation and Cooperative Calcium Contraction of Glycerinated Vorticella1, The Journal of Biochemistry, Vol.83, No.3 (1978), pp.795-798.

Carlsen, R. W. and Sitti, M., Bio-Hybrid Cell-Based Actuators for Microsystems, Small, Vol.10, No.19 (2014), pp.3831-3851.

Cheng, Y., Luo, X., Betz, J., Payne, G. F., Bentley, W. E. and Rubloff, G. W., Mechanism of anodic electrodeposition of calcium alginate, Soft Matter, Vol.7, No.12 (2011a), pp.5677-5684.

Cheng, Y., Luo, X., Tsao, C.-Y., Wu, H.-C., Betz, J., Payne, G. F., Bentley, W. E. and Rubloff, G. W., Biocompatible multiaddress $3 \mathrm{D}$ cell assembly in microfluidic devices using spatially programmable gel formation, Lab on a Chip, Vol.11, No.14 (2011b), pp.2316-2318.

Di Carlo, D., Ionescu-Zanetti, C., Zhang, Y., Hung, P. and Lee, L. P., On-chip cell lysis by local hydroxide generation, Lab on a Chip, Vol.5, No.2 (2005), pp.171-178.

Din, M. O., Danino, T., Prindle, A., Skalak, M., Selimkhanov, J., Allen, K., Julio, E., Atolia, E., Tsimring, L. S., Bhatia, S. N. and Hasty, J., Synchronized cycles of bacterial lysis for in vivo delivery, Nature, Vol.536, No.7614 (2016), pp.81-85.

Haubert, K., Drier, T. and Beebe, D., PDMS bonding by means of a portable, low-cost corona system, Lab on a Chip, Vol.6, No.12 (2006), pp.1548-1549.

Hümmer, D., Kurth, F., Naredi-Rainer, N. and Dittrich, P. S., Single cells in confined volumes: microchambers and microdroplets, Lab on a Chip, Vol.16, No.3 (2016), pp.447-458.

Jamur, M. C. and Oliver, C., 2010, Immunocytochemical Methods and Protocols, Humana Press, Totowa, NJ, Permeabilization of Cell Membranes.

Katoh, K. and Naitoh, Y., Control of Cellular Contraction by Calcium in Vorticella, The Journal of Experimental Biology, Vol.189, No.1 (1994), pp.163-177.

Lee, H. J., Kim, J. H., Lim, H. K., Cho, E. C., Huh, N., Ko, C., Park, J. C., Choi, J. W. and Lee, S. S., Electrochemical cell lysis device for DNA extraction, Lab on a Chip, Vol.10, No.5 (2010), pp.626-633.

Magdanz, V., Sanchez, S. and Schmidt, O. G., Development of a Sperm-Flagella Driven Micro-Bio-Robot, Advanced Materials, Vol.25, No.45 (2013), pp.6581-6588.

Nagai, M., Ryu, S., Thorsen, T., Matsudaira, P. and Fujita, H., Chemical control of Vorticella bioactuator using microfluidics, Lab on a Chip, Vol.10, No.12 (2010), pp.1574-1578.

Nagai, M., Hayasaka, Y., Kato, K., Kawashima, T. and Shibata, T., Mixing of solutions by coordinated ciliary motion in Vorticella convallaria and patterning method for microfluidic applications, Sensors and Actuators B: Chemical, Vol.188 (2013a), pp.1255-1262.

Nagai, M., Matsumoto, N., Kawashima, T. and Shibata, T., Reversible motion control of Vorticella stalk in microchannel, Microelectronic Engineering, Vol.108 (2013b), pp.28-32.

Nagai, M., Oguri, M. and Shibata, T., Characterization of light-controlled Volvox as movable microvalve element assembled in multilayer microfluidic device, Japanese Journal of Applied Physics, Vol.54, No.6 (2015), pp.067001.

Nagai, M., Tanizaki, K. and Shibata, T., Batch Assembly of SU-8 Movable Components in Channel Under Mild Conditions for Dynamic Microsystems: Application to Biohybrid Systems, Journal of Microelectromechanical Systems, Vol.28, No.3 (2019a), pp.419-428.

Nagai, M., Tanizaki, K. and Shibata, T., Batch Assembly of SU-8 Movable Components in Channel Under Mild Conditions for Dynamic Microsystems:Application to Biohybrid Systems, Journal of Microelectromechanical Systems, Vol.28, No.3 (2019b), pp.419-428.

Nevill, J. T., Cooper, R., Dueck, M., Breslauer, D. N. and Lee, L. P., Integrated microfluidic cell culture and lysis on a chip, Lab on a Chip, Vol.7, No.12 (2007), pp.1689-1695.

Nicolau, D. V., Lard, M., Korten, T., Van Delft, F. C. M. J. M., Persson, M., Bengtsson, E., Månsson, A., Diez, S., Linke, H. and Nicolau, D. V., Parallel computation with molecular-motor-propelled agents in nanofabricated networks, Proceedings of the National Academy of Sciences, Vol.113, No.10 (2016), pp.2591-2596.

Ochiai, T., Asai, H. and Fukui, K., Hysteresis of Contraction-Extension Cycle of Glycerinated Vorticella, The Journal of 
Protozoology, Vol.26, No.3 (1979), pp.420-425.

Ryu, S., Pepper, R. E., Nagai, M. and France, D. C., Vorticella: A Protozoan for Bio-Inspired Engineering, Micromachines, Vol.8, No.1 (2017), pp.4.

Shehadul Islam, M., Aryasomayajula, A. and Selvaganapathy, P. R., A Review on Macroscale and Microscale Cell Lysis Methods, Micromachines, Vol.8, No.3 (2017), pp.83.

Upadhyaya, A., Baraban, M., Wong, J., Matsudaira, P., Van Oudenaarden, A. and Mahadevan, L., Power-Limited Contraction Dynamics of Vorticella convallaria: An Ultrafast Biological Spring, Biophysical Journal, Vol.94, No.1 (2008), pp.265272.

Vacchiano, E., Kut, J., Wyatt, M. and Buhse, H., A novel method for mass-culturing Vorticella, The Journal of protozoology, Vol.38, No.6 (1991), pp.609-613.

Zhang, W. Y., Ferguson, G. S. and Tatic-Lucic, S., Elastomer-supported cold welding for room temperature wafer-level bonding, 17th IEEE International Conference on Micro Electro Mechanical Systems. Maastricht MEMS 2004 Technical Digest, (2004), pp.741-744. 